\title{
Use of IFNy/IL10 ratio for stratification of hydrocortisone therapy in patients with septic shock
}

Rainer König ${ }^{1,2,+, *}$, Amol Kolte ${ }^{1,2,{ }^{*}}$, Olaf Ahlers ${ }^{3 *}$, Marcus Oswald ${ }^{1,2, *}$, Daniela Röll ${ }^{1,2}$, George Dimopoulos $^{4}$, Iraklis Tsangaris ${ }^{4}$, Eleni Antoniadou ${ }^{5}$, Holger Bogatsch ${ }^{6}$, Markus Löffler ${ }^{7}$, Charles

L. Sprung ${ }^{8}$, Mervyn Singer ${ }^{9}$, Frank Brunkhorst ${ }^{1}$, Michael Oppert ${ }^{10}$, Herwig Gerlach ${ }^{11}$, Ralf A. Claus $^{12}$, Sina M. Coldewey ${ }^{12,13,1}$, Josef Briege ${ }^{14}$, Evangelos J. Giamarellos-Bourboulis ${ }^{15,1}$, Didier Keh" ${ }^{3, \#}$ Michael Bauer ${ }^{1,12,+, \#}$

${ }^{1}$ Integrated Research and Treatment Center Center for Sepsis Control and Care (CSCC), Jena University Hospital, Germany

${ }^{2}$ Network Modeling, Leibniz Institute for Natural Product Research and Infection Biology Hans Knöll Institute Jena, Jena, Germany.

${ }^{3}$ Department of Anesthesiology and Operative Intensive Care Medicine (CCM, CVK), Charité - Universitätsmedizin Berlin, corporate member of Freie Universität Berlin, HumboldtUniversität zu Berlin, and Berlin Institute of Health; Campus Virchow-Klinikum, Berlin, Germany

${ }^{4}$ 2nd Department of Critical Care Medicine, National and Kapodistrian University of Athens, Medical School, Greece

${ }^{5}$ Intensive Care Unit, "George Gennimatas” Thessaloniki General Hospital, Greece

${ }^{6}$ Clinical Trial Centre, Leipzig University, Leipzig, Germany

${ }^{7}$ Institute for Medical Informatics, Statistics and Epidemiology (IMISE), Leipzig University, Leipzig, Germany

${ }^{8}$ Department of Anesthesiology and Critical Care Medicine, Hadassah Hebrew University Medical Center, Jerusalem, Israel

${ }^{9}$ Bloomsbury Institute of Intensive Care Medicine, Division of Medicine, University College London, Gower St, London WC1E 6BT, UK

${ }^{10}$ Klinikum Ernst von Bergmann, Potsdam, Germany

${ }^{11}$ Department of Anesthesia, Operative Intensive Care Medicine, and Pain Management, Vivantes Neukölln Hospital, Berlin, Germany

${ }^{12}$ Department of Anesthesiology and Intensive Care Medicine, Jena University Hospital, Jena, Germany

${ }^{13}$ Septomics Research Center, Jena University Hospital, Jena, Germany 
${ }^{14}$ Department of Anesthesiology, University Hospital, LMU Munich, Germany

15 4th Department of Internal Medicine, National and Kapodistrian University of Athens, Medical School, Greece

*Equal contribution, \# Equal contribution

+ Correspondence: rainer.koenig@uni-jena.de and michael.bauer@uni-jena.de 


\section{Abstract}

Background: Large clinical trials testing hydrocortisone therapy in septic shock have produced conflicting results. Subgroups may however benefit depending on their individual immune response.

Methods: We performed an exploratory analysis of the CORTICUS trial database employing machine learning to a panel of 137 variables collected from 83 patients (60 survivors, 23 non-survivors) including demographic and clinical measures, organ failure scores, leukocyte counts and circulating cytokine levels. The identified biomarker was validated against data collected from patients enrolled into a cohort of the Hellenic Sepsis Study Group (HSSG) $(n=162)$ and two data sets of two other clinical trials. Ex vivo studies were performed on this biomarker to assess a possible mechanistic role.

Results: A low serum IFNY/L10 ratio predicted increased survival in the hydrocortisone group whereas a high ratio predicted better survival in the placebo group. Using this ratio for a decision rule, we found significant improvement in survival in the groups of patients being in compliance with the prediction rule (discovery set: $\mathrm{OR}=3.03$ [95\% $\mathrm{Cl}: 1.05-8.75], \mathrm{P}=0.031$, validation set: $\mathrm{OR}=2.01$ [95\% Cl: 1.04-3.88], $\mathrm{P}=0.026$ ). Applying the rule to two further, smaller datasets showed the same tendency. Mechanistic studies revealed that IFNY/IL10 was negatively associated with pathogen load in spiked human blood. An in silico analysis of published IFNy and IL10 values in bacteremic and non-bacteremic SIRS patients supported this association between the ratio and pathogen burden.

Conclusion: If confirmed prospectively, the IFNY/LL10 ratio could be used as a rapidly available theranostic for use of hydrocortisone therapy in septic shock.

Key words: sepsis, hydrocortisone, immune therapy, machine learning, theranostics, IFNy/IL10 ratio 


\section{Introduction}

Though prospective, randomized, controlled multicentre trials have consistently reported faster shock resolution $(1,2)$, the utility of 'low-dose' hydrocortisone $(\mathrm{HC})$ in patients with septic shock remains controversial. Whereas two French studies reported outcome benefit from a combination of hydrocortisone plus oral fludrocortisone $(3,4)$, the pan-European CORTICUS trial and the 5-country ADRENAL trial found no survival effect from hydrocortisone alone $(2,5)$. Possible explanations for this disparity included differences in mortality risk in the populations with a two-fold higher risk of mortality in the French control group (of (1)) compared to CORTICUS (61\% versus $31 \%$, respectively), and an increase in superinfections, variations in other aspects of clinical management, and genetic variations. Of note, a subset analysis of the ADRENAL trial indicated survival benefit from hydrocortisone in Australasian patients, no effect in British and Danish patients, and a trend to harm in patients enrolled in Saudi Arabia (2).

It is increasingly recognized that patients presenting in septic shock are hyper-inflamed yet at the same time immunosuppressed (6-8). Corticosteroids are traditionally considered to induce immune suppression via the glucocorticoid receptor $(G R)$ and its repressive effect on pro-inflammatory transcription factors such as AP-1 and NFKB (9). Thus, patients in an overall state of immunosuppression may be potentially compromised by administration of an immunosuppressive drug. This argument is however complicated by an increasing evidence base implicating corticosteroids and GRs in immune-reconstitutive processes $(10,11)$. In human monocytes, corticosteroid treatment induced expression of innate immune-related genes, such as TLRs, and anti-inflammatory genes $(10,11)$. In macrophages and derived cell lines glucocorticoids induced a central component of the inflammasome (NLRP3) and, upon stimulation with endotoxin, induced secretion of pro-inflammatory cytokines such as tumor necrosis factor alpha (TNFa) (11). Furthermore, glucocorticoid-dependent NLRP3 induction resulted in sensitization of innate immune cells to extracellular ATP and thus an enhanced ATP-mediated secretion of pro-inflammatory cytokines following endotoxin 
stimulation (12). This immune-activating role of corticosteroids has been described as a response to acute stress enhancing the peripheral immune response, whereas chronic corticosteroid exposure leads to immune suppression $(13,14)$. These diverging effects of GCs support the need for biomarkers to guide their application. We applied machine learning to physiological and laboratory data from patients enrolled into a CORTICUS sub-study to determine a theranostic marker for hydrocortisone treatment. We found the ratio of serum interferon-y (IFNY) to interleukin-10 (IL-10) to identify specific sub-cohorts with increased and decreased survival upon treatment. We thus explored this predictive utility of this biomarker in further datasets of septic shock patients and performed in vitro mechanistic studies to explore the possible significance of this ratio.

\section{Materials and Methods}

\section{The CORTICUS cohort}

In addition to the standard CORTICUS protocol, the Berlin study group sampled blood for subsequent measurement of cytokines and other circulating inflammatory mediators from 84 patients in 13 participating sites. The study was approved by the local Ethics Committee (no: 153/2001). Written informed consent was obtained from patients, proxies or their legal representatives. Eligible patients were enrolled if they met the following inclusion criteria: clinical evidence of infection, evidence of a systemic response to infection, the onset of shock within the previous 72 hours and hypoperfusion or organ dysfunction attributable to sepsis. Notable exclusion criteria included an underlying disease process with a poor prognosis, life expectancy $<24$ hours, long-term immunosuppression, and treatment with long-term corticosteroids within the past 6 months or short-term corticosteroids within the past 4 weeks. Detailed eligibility criteria are given in Supplementary Table S1 and the original study (5). Patients were randomized to receive either placebo or $200 \mathrm{mg}$ hydrocortisone $(\mathrm{HC})$ /day for 5 days, followed by a tapering dose until day 11 . Demographic and baseline characteristics were extracted from the CORTICUS database. 79 out of 83 
(95\%) patients received norepinephrine at baseline while none received epinephrine. Blood samples were taken directly before an ACTH stimulation test and administration of the study medication. Table S2 shows the timing of blood sampling relative to the onset of shock. Blood samples were collected on day 0 , on day 2, on the morning of day 5 (end of full dose HC application), on day 12 (day after HC cessation), day 17 and 27 . The soluble mediators interleukin-(IL)-6, -8, -10, -12 p70, IFNy, TNFa, soluble TNF-receptor-I (sTNF-RI), soluble FAS (all OptEIA ( ${ }^{\mathrm{TM}}$ ) Set Human, BD Biosciences, New Jersey, USA), and E-selectin (R\&D, Minnesota, USA) were measured in serum, plasma, or culture supernatant by enzyme-linked immunosorbent assay (ELISA) according to the manufacturers' instructions. This included calculating calibration and standard curves. All measurements were performed in duplicate. The cytokine and all other laboratory values were taken from the original study.

One patient was removed due to lack of cytokine data. For ten patients either IFNY or IL10 were below the detection limit. Excluding these patients did not change the overall results (Supplementary Text S8). Of the remaining 83 patients, serum lactate values (pre-treatment) and temporal values were available in 53 and 41 patients, respectively. For full details, see Supplementary Text S1 and S2.

\section{The Hellenic Sepsis Study Group (HSSG) cohort and two further cohorts for validation} Validation was performed on data obtained by the Hellenic Sepsis Study Group from septic shock patients with community-acquired pneumonia or intraabdominal infection. This study included a prospective collection of clinical data and biosamples from patients admitted to 45 study sites in Greece. Patients were enrolled after written informed consent provided by themselves or their legal representatives. Detailed eligibility criteria are given in Supplementary Table S1. All enrolled patients had been reclassified into infection and sepsis using the Sepsis-3 classification criteria $(15,16)$. In HC-treated patients $200 \mathrm{mg} /$ day HC had been administered for 7 days followed by gradual tapering. Secreted cytokines were measured using the LEGENDplex Human Inflammation Panel (13-plex) (BioLegend, San 
Diego, USA) according to the manufacturer's instructions with half of the reagents volume and sample incubation at $4^{\circ} \mathrm{C}$ overnight. After quality control and discarding data from patients dying or being discharged on the day of admission, a total of 162 eligible shock patients (HC treatment: $n=63$, No HC treatment: $n=99$ ) were selected. If only one of the cytokines (IFNy or IL10) was below the detection limit, the respective value was set as the detection limit. Excluding these samples from the analysis did not alter the findings (Supplementary Text S8). For further details, see Supplementary Text S3 and Table S3 for patient characteristics. Furthermore, we investigated serum IFNY/L10 of patients in the placebo arm of the randomized placebo-controlled, trial of Sodium Selenite and Procalcitonin-guided antimicrobial therapy in Severe Sepsis (SISPCT) (17). After propensity score matching, $\mathrm{n}=24$ patients were included in the analysis (details about the study and statistics, see Supplementary Text S6). In addition, we analyzed serum IFNY/IL10 of patients from an earlier small crossover study (18) (details about this study and the crossover scheme is given in Supplementary Text S7). In this study, the early arm got a comparable HC application as the HC arm of CORTICUS, and hence was also used for validating our marker. The cytokine values of the crossover study were taken from the original publication.

\section{Ex vivo whole blood culture experiments}

To assess biological plausibility of the data-driven biomarker IFNY/IL10, we performed ex vivo whole blood culture experiments in which blood of healthy donors was spiked with a broad range of bacterial products to simulate pathogen load. $200 \mu \mathrm{L}$ of diluted (HBSS (1:1, V/V) heparinized whole blood obtained from healthy volunteers ( $n=5$, male, 20-25 years) was stimulated with serial dilutions either of LPS (endotoxin of E. coli B4:0111) (Sigma Aldrich) or lysates from two E. coli isolates obtained from septic patients). E. coli lysates were created by sonification, following heat inactivation, and a serial dilution of the obtained fragment stock was performed. Following incubation $\left(37^{\circ} \mathrm{C}, 18\right.$ hours, gentle agitation at $\left.2 \mathrm{rpm}\right)$ plasma supernatant was prepared by centrifugation $(2500 \mathrm{~g}$ at room temperature for $10 \mathrm{~min}$ ), 
secreted cytokine levels were measured using the LEGENDplex Human Inflammation Panel (13-plex) (BioLegend), as described above. Comparison was made to vehicle controls.

\section{The rationale of the data analysis}

We used the database consisting of 137 patient features (potential predictors) and 83 patients from the subcohort of the CORTICUS study population to perform an exploratory data analysis. This table also included the ratios of all cytokine combinations. We aimed to find a theranostic marker distinguishing $\mathrm{HC}$ responders (i.e. survivors) from non-responders (non-survivors). The complete list of available features is shown in Supplementary Table S4 and the workflow depicted in Supplementary Figure S1.

We sought the best predictor of 28-day survival for the placebo arm using one-level decision trees (stumps) calculated by a leave-one-out cross-validation scheme. The best decision trees were chosen by intelligent enumeration. Table S5 lists the results from all 41 crossvalidation runs. Of these, the predictor and threshold, which was in most trees (i.e. the predictor IFNy/IL10) was applied to the HC arm. In 39 out of 41 runs, the cutoff value was 0.95 and applied to the $\mathrm{HC}$ arm leading to the 39.8 percentile of all 83 samples. Patients whose IFNy/IL10 ratio ranked among the first $39.8 \%$ patients were denoted "low-ratio patients", the others "high-ratio patients". When we weighted all non-surviving patients higher or lower, we observed a linear relation between the optimal percentile and the corresponding mortality rates. Hence, we calculated these optimal cutoffs to gain a calibration curve with which we determined the cutoff for the validation sets (see also Results and Discussion, details for the method are in Supplementary Text S2, Figure S2). All analyses were carried out in R (www.r-project.org) using custom scripts. Odds ratio and statistical significance calculations are described in Supplementary Text S2. 


\section{Ex vivo whole blood culture experiments}

To assess biological plausibility of the data-driven biomarker IFNY/IL10, we performed ex vivo whole blood culture experiments in which blood of healthy donors was spiked with a broad range of bacterial products to simulate pathogen load. $200 \mu \mathrm{L}$ of diluted (HBSS (1:1, V/V) heparinized whole blood obtained from healthy volunteers ( $n=5$, male, 20-25 years) was stimulated with serial dilutions either of LPS (bacterial endotoxin of E. coli B4:O111) (Sigma Aldrich) or lysates from two E. coli isolates obtained from septic patients). E. coli lysates were created by sonification, following heat inactivation, and a serial dilution of the obtained fragments stock was performed. Following exposition $\left(37^{\circ} \mathrm{C}, 18\right.$ hours, gently agitation $\left.2 \mathrm{rpm}\right)$ plasma supernatant was prepared by centrifugation $(2.500 \mathrm{~g}, \mathrm{RT}, 10 \mathrm{~min})$, secreted cytokine levels were measured using the LEGENDplex Human Inflammation Panel (13-plex) (BioLegend) as described above compared to vehicle control.

\section{Results}

\section{Patient characteristics}

Patient characteristics are summarized in Table 1. Median plasma concentrations of soluble mediators and leukocytes were not significantly different between the arms at baseline (Supplementary Table S6). Age differed but was not a confounder (see Supplementary Text S4).

\section{IFNy/IL10 stratifies CORTICUS patients}

Analysis of baseline characteristics was performed on 137 variables including demographic and clinical variables, Sepsis-related Organ Failure Assessment (SOFA) scores, lymphocyte counts, plasma protein concentrations of cytokines and patient blood stimulation experiments (Supplementary Table S4). We performed a leave-one-out cross-validation with one-level decision trees (using only one predictor at a time) to the placebo arm to discriminate between 28-day-survivors and non-survivors. This led to a high true positive rate (83\%, Table $2 a)$. In 
$95 \%$ of the cross-validation runs, the serum IFNY/IL10 ratio (referred to as IFNY/LL10 in the following) with the same threshold (39.8 percentile of IFNY/IL10 from all patients) was selected by the algorithm as the best predictor. Patients which IFNY/IL10 ratios ranked among the first $39.8 \%$ patients were denoted "low-ratio patients", the others "high-ratio patients". Upon applying this predictor to HC-treated patients, the reverse behaviour was observed with a high true negative rate i.e. a low IFNy/IL10 indicated a high likelihood of survival $(85 \%)$ (Table $2 b)$. A significant interaction effect $(p=0.0083)$ was observed between the IFNY/L10 ratio and the treatment (Supplementary Text S11, Figure S3). These observations were then compiled into a decision rule: no $\mathrm{HC}$ treatment if the ratio was high, and $\mathrm{HC}$ treatment if low. This decision rule yielded an odds ratio (OR) of survival of 3.03 [95\% Cl: 1.05-8.75], $\mathrm{p}=0.031$. Of note, neither IFNy nor IL10 alone could allow this prediction. Our new treatment rule was applied to a validation dataset from the Hellenic Sepsis Study Group and to two smaller datasets from two other clinical trials.

\section{Validation based on patients from the Hellenic Sepsis Study Group (HSSG) and two further datasets}

Table S3 summarizes demographics of the validation cohort. Applying IFNY/IL10 to the group of HSSG patients, we observed a similar pattern as for the CORTICUS subgroup. High IFNY/IL10 indicated distinct higher survival of the HC non-treated patients (50\% versus 19\%). In contrast, in the $\mathrm{HC}$ treated group we observed the opposite behaviour (28\% versus $35 \%$ ), yielding an odds ratio of $\mathrm{OR}=2.01$ [95\% Cl: 1.04-3.88], $\mathrm{P}=0.026$. As the survival rate of the HSSG patients was lower compared to CORTICUS, we had to adjust the threshold according to a calibration scheme based on the CORTICUS data explained in Methods. Figure 1 illustrates the potential of the theranostic marker to predict responders in both cohorts. Adjusting for an imbalance in co-morbidities between $\mathrm{HC}$ treated and non-treated patients by propensity score matching led to the same result (Supplementary Text S3). 
Furthermore, we investigated serum IFNY/IL10 of patients in 24 (propensity score matched) patients from the placebo arm of the randomized placebo-controlled, trial of Sodium Selenite and Procalcitonin-guided antimicrobial therapy in Severe Sepsis (SISPCT) (17) (details about the study and statistics, see Supplementary Text S6). All patients survived $(n=3)$ if treated according to the rule, compared to $77 \%$ if not treated according to the rule. In addition, we analyzed serum IFNy/IL10 of patients from an earlier small crossover study (18) in which the early arm got a comparable HC application as the HC arm of CORTICUS, and hence was also used for validating our marker (details about this study and the crossover scheme is given in Supplementary Text S7). In line to the results from CORTICUS, HSSG and SISPCT, low IFNy/IL10 indicated good survival (88\% survivors), whereas high IFN/IL10 was an indicator for considerably worse outcome ( $57 \%$ survivors), for $\mathrm{HC}$ treatment the survival rates between IFNy/IL10 high and low were comparable (Table 2e). Due to the small sample sizes the results from SISPCT and the crossover study failed to achieve significance. In summary, the investigated patients from all studies evidenced IFNY/IL10 as a potential theranostic marker for HC application in septic shock.

\section{Time courses of serum lactate and norepinephrine requirement reflect hemodynamic} stabilization in patients treated in compliance with the decision rule

High serum lactate levels have been demonstrated to indicate severity of metabolic derangements and increased mortality in sepsis (15). Median initial lactate was $1.89 \mathrm{mmol} / \mathrm{L}$ in patients with high compared to $2.89 \mathrm{mmol} / \mathrm{L}$ in patients with low IFNY/IL10 suggesting that IFNY/IL10 associated with disease severity (boxplots, see Supplementary Figure S4). Of note, although the lactate levels correlate inversely with IFNy/IL10 at baseline, serum lactate itself performed worse as a theranostic marker. Notably, we identified significant lactate decrease specifically in the group of patients, which were treated in compliance with the decision rule (Figure 2 a-d). Hemodynamic stabilization was also supported by the reduction of norepinephrine $(N E)$ requirement $(P=2.0 e-05)$ specifically in the group of patients in 
compliance with the decision rule (Figure 2 e-h). In summary, time series of serum lactate and NE requirement reflect better recovery of septic shock in patients treated in compliance with the proposed theranostic marker.

\section{IFNy/IL10 as an indicator of pathogen load}

In light of published evidence associating IFNy and IL10 with the severity of parasitic and tuberculosis infection, we investigated whether IFNY/LL10 reflects the pathogen burden of immune cells when challenged with typical pathogens found in sepsis. We spiked blood from healthy donors with $E$. coli fragments from clinical isolates or endotoxin across a wide range of concentrations, mimicking the immunological load. As expected, the higher the load, the higher the immune response and hence the concentration of IFNY and IL10 in the supernatant (Figure 3a, b). Remarkably, we observed the inverse behaviour for the ratio, i.e. a high IFNY/L10 was observed for low pathogen concentrations and vice versa with "on-off" kinetics (Figure 3c). Results for a second E. coli isolate or endotoxin were similar (Supplementary Figure S5). To associate IFNy/IL10 to the bacterial load in critical ill patients, we compared publically available data of patients with and without bacteremia. Matera et al. (19) investigated 52 patients (39 survivors and 13 non-survivors) with signs of SIRS at hospital admission. Only $4 \%$ of them were in septic shock. 28 of 52 were diagnosed with bacteremia. In line with our ex vivo observations, a distinctively higher ratio $(1.6 \pm 0.77)$ for IFN/IL10 in SIRS patients was observed compared to patients with bacteremia $(0.8 \pm 0.88$, details: Supplementary Text S9). Healthy volunteers showed the highest IFNy/LL10 (2.80 \pm 1.22) of all groups investigated by Matera et al.. In summary, IFNY/IL10 inversely correlates with the immunological load of infection in an in vitro system and when comparing bacteremic and non-bacteremic critically ill patients. 


\section{The corticotropin test and IFNy/IL10 correlation to the severity of septic shock}

Poor response to corticotropin stimulation is no longer recommended for HC treatment (3). We tried several machine learning concepts to stratify for treatment using serum HC baseline and after corticotropin stimulation. In line to results of the original study (5), the corticotropin test failed to predict response to treatment (Figure 1b, Supplementary Text S10, Table S7). We further investigated if IFNy/IL10 correlates with the severity of septic shock. However, IFNY/IL10 did neither correlate with mean arterial pressure nor with the SOFA score in the investigated CORTICUS cohort.

\section{Discussion}

Starting with a larger panel of predictors from septic shock patients treated with $\mathrm{HC}$ or placebo in CORTICUS, we identified the ratio of serum IFNY and IL10 as a promising biomarker for $\mathrm{HC}$ treatment decision. The decision rule was based on data derived from 83 patients. We validated our results by applying the marker and threshold to three other, unseen datasets to validate the potential of the theranostic marker. As in particular the survival rate of the HSSG cohort was very different to CORTICUS, this threshold needed to be adjusted. This was performed by a calibration curve calculated from the CORTICUS dataset mimicking higher death rates in CORTICUS. This led to a positive correlation between the optimal threshold and the mimicked death rate (details, see Supplementary Text S2), i.e. the higher the threshold, the more patients are in a worse condition at baseline (higher incidence of death), and particular these patients were observed to benefit from $\mathrm{HC}$. Albeit the ratio was identified by machine learning and supported by subsequent in vitro experiments, there is published evidence for its plausibility as a biomarker for life-threatening infections. IFNy and IL10 have been suggested as biomarkers for parasitic (20) and other infections, such as tuberculosis (21). Mcllleron et al. reported the time course of IFNY indicating response to treatment in pulmonary tuberculosis (22). However, for diagnosis of pulmonary tuberculosis, IFNy alone has limitations with relation to sensitivity (23). In turn, 
high IL10 was observed with mycobacterial persistence (24) suggesting that in particular the ratio of these cytokines might reflect the immunological burden of infection. In line, it has been shown that the ratio of IFNy and IL10 correlates with the disease severity of tuberculosis and may differentiate pulmonary from extrapulmonary forms (25). To elucidate the functional implications of low and high IFNy/IL10, we performed blood culture assays. Indeed, we observed the ratio to be associated with the pathogen load in ex vivo blood culture experiments. This was supported by data from Matera et al. (19) when regarding critically ill patients with bacteremia (higher bacterial load) and without bacteremia (no or non-detectable bacterial load).

Synoptically, IFNY/IL10 is associated with pathogen load and thus, severity of sepsis. Metabolically, high serum lactate indicates severity of cellular disturbances (15) and is associated with poor outcome (26). Consistent with this, patients with low IFNY/IL10 showed high serum lactate. Furthermore, stratifying patients in compliance with the proposed decision rule, we observed a considerable decrease over time in serum lactate specifically in the group of patients, which were treated in compliance with the theranostic marker.

IFNy has been consistently documented to activate cells of adaptive immunity (27) and IL10 as a suppressor of innate immune responses and inflammation $(28,29)$. According to this, high IFNY/IL10 reflects increased activated adaptive and innate immunity. In line, we observed ex vivo that blood challenged with bacterial fragments or LPS, displayed low IFNY/IL10 at high loads, and vice versa. Transferring these observations to septic shock patients with low IFNy/IL10, we speculate that HC treatment yielded a better outcome in HCresponders because HC may allow the highly loaded immune system more pace for recovery. This is evidenced, by the reduction of serum lactate and norepinephrine requirements as measures of recovery particularly in the subgroup of very sick patients.

The concern about side effects of corticosteroids such as infections in patients with less severe septic shock stipulated more restrictive recommendations by the Surviving Sepsis Campaign. $\mathrm{HC}$ application is currently recommended for patients not responding to adequate 
fluid resuscitation and vasopressor therapy $(30,31)$. It seems intuitively promising to include an immune biomarker, such as IFNy/IL10 reflecting the status of the patients' immune system. Consistent with this concept, Bentzer et al. studied patients from the VASST trial investigating corticosteroid treated versus non-treated (only vasopressin or catecholamine vasopressors). They identified a signature of three cytokines (IL3, IL6, CCL4) suggesting response to corticosteroid treatment (32). However, these results are based on a study, which was not randomized, blinded or protocolized according to corticosteroid treatment. Further, Bentzer et al. did not distinguish between vasopressin and catecholamine treatment, and they didn't elaborate explaining how these three cytokines interact with corticosteroid treatment.

Applying our decision rule successfully across a broad range of studies suggests the marker to be generic and rather independent from specific assays, probably reflecting the on-off kinetics of the quotient.

\section{Limitations and strengths}

The relatively small sample sizes along with the high dimensional data sets obtained imply that our results must be interpreted with caution and requires prospective validation. A further limitation of our study was that the use of corticosteroids was not randomized in the HSSG and the SISPCT study. Strengths of our study were that we used well-phenotyped cohorts of patients with septic shock, that our marker showed very similar results across all studies, and that we not only showed the potential clinical relevance but also give a reasonable functional reasoning why this marker may support $\mathrm{HC}$ application by investigating ex vivo blood culture experiments. 


\section{Conclusions}

We identified the ratio of serum cytokines IFNY and IL10 as a theranostic marker for hydrocortisone treatment in septic shock. An accompanying study into the mechanism suggests that this ratio enables the host to sense the pathogen load in an "on-off" fashion.

\section{Abbreviations}

ACTH: Adrenocorticotropic hormone

ADRENAL: Adjunctive corticosteroid treatment in critically ill patients with septic shock

Cl: Confidence interval

CORTICUS: Corticosteroid therapy of septic shock

HC: Hydrocortisone

HSSG: Hellenic sepsis study group

NE: Norepinephrine

OR: Odds ratio

SISPCT: Sodium selenite and procalcitonin guided antimicrobial therapy in severe sepsis

SOFA: Sequential organ failure assessment

VASST: Vasopressin and septic shock trial

\section{Declarations}

\section{Acknowledgements}

We thank Anne Goessinger for her outstanding support of the CORTICUS sub-study.

\section{Funding}

This work has been supported by the Federal Ministry of Education and Research (BMBF), Germany, FKZ: 01E01002, $01 E 01502$ (CSCC), and FKZ 01ZX1302B, 01ZX1602B (CancerTel-Sys), 03Z22JN12 (Translational Septomics) and department funds of the 
Department of Anesthesiology and Intensive Care Medicine, Jena University Hospital. The CORTICUS immune-substudy was supported by the German Research Foundation (Deutsche Forschungsgemeinschaft, DFG): KE 870 1-1 and 1-2., and by department funds of the Department of Anesthesiology and Operative Intensive Care Medicine (CCM, CVK), Charité - Universitätsmedizin Berlin.

\section{Availability of data and materials}

Most of the data is provided in the main text or supplementary material. The other datasets used or analyzed are available from the corresponding author on reasonable request.

\section{Authors' contributions}

RK, AK, MO, MB conceptualized and designed the study. MO, RK and AK developed the methodology. AK, MB, MS, JB, DK, DR and RK analyzed and interpreted the data. Administrative, technical, or material support was given by OA, DR, GD, IT, EA, HB, ML, CLS, MS, FB, MO, HG, RAC, SMC and JB. The study was supervised by RK, DK and MB. All authors read and approved the final manuscript.

\section{Ethics approval and consent to participate}

The CORTICUS trial was a multicenter study, the protocol was approved by the ethics committee at each of the 52 participating intensive care units. In addition to the standard CORTICUS protocol, the Berlin study group sampled blood for subsequent measurement of cytokines and other circulating inflammatory mediators. This was approved by the local ethics committee (no: 153/2001). Written informed consent was obtained from patients, proxies or their legal representatives. The HSSG cohort represents a prospective collection of clinical data and biosamples in 45 study sites in Greece. The study protocol was approved from the ethics committees of all participating hospitals. Patients were enrolled after written informed consent provided by themselves or by first-degree relatives if patients were unable 
to consent. The Placebo-Controlled Trial of Sodium Selenite and Procalcitonin Guided Antimicrobial Therapy in Severe Sepsis (SISPCT) was a multicenter clinical trial. It was conducted in 33 multidisciplinary intensive care units across Germany. The study protocol was approved by the ethics board of Jena University Hospital. Written informed consent was obtained from all patients or their legal representatives. Among these, 109 patients were included in the Munich (Ludwig-Maximilians-University, LMU) sub-study for which cytokine measurements for this study was performed using their blood samples (ethics votum amendment EudraCT: 2007-004333-42). The study protocol for the Crossover study was approved by the institutional (Charite, Berlin) ethics committee.

\section{Consent for publication}

Not applicable.

\section{Competing interests}

The authors declare that they have no competing interests.

\section{References}

1. Annane D, Bellissant E, Bollaert P-E, Briegel J, Confalonieri M, De Gaudio R, Keh D, Kupfer Y, Oppert M, Meduri GU. Corticosteroids in the Treatment of Severe Sepsis and Septic Shock in Adults. JAMA 2009;301:2362.

2. Venkatesh B, Finfer S, Cohen J, Rajbhandari D, Arabi Y, Bellomo R, Billot L, Correa M, Glass P, Harward M, Joyce C, Li Q, McArthur C, Perner A, Rhodes A, Thompson K, Webb S, Myburgh J, ADRENAL Trial Investigators and the Australian-New Zealand Intensive Care Society Clinical Trials Group. Adjunctive Glucocorticoid Therapy in Patients with Septic Shock. N Engl J Med 2018;378:797-808.

3. Annane D, Sébille V, Charpentier C, Bollaert P-E, François B, Korach J-M, Capellier G, Cohen Y, Azoulay E, Troché G, Chaumet-Riffaud P, ChaumetRiffaut $\mathrm{P}$, Bellissant $\mathrm{E}$. Effect of treatment with low doses of hydrocortisone and fludrocortisone on mortality in patients with septic shock. JAMA 2002;288:862871.

4. Annane D, Renault A, Brun-Buisson C, Megarbane B, Quenot J-P, Siami S, Cariou A, Forceville X, Schwebel C, Martin C, Timsit J-F, Misset B, Ali Benali M, Colin G, Souweine B, Asehnoune K, Mercier E, Chimot L, Charpentier C, 
François B, Boulain T, Petitpas F, Constantin J-M, Dhonneur G, Baudin F, Combes A, Bohé J, Loriferne J-F, Amathieu R, et al. Hydrocortisone plus Fludrocortisone for Adults with Septic Shock. N Engl J Med 2018;378:809-818.

5. Sprung CL, Annane D, Keh D, Moreno R, Singer M, Freivogel K, Weiss YG, Benbenishty J, Kalenka A, Forst H, Laterre P-F, Reinhart K, Cuthbertson BH, Payen D, Briegel J. Hydrocortisone Therapy for Patients with Septic Shock. $N$ Engl J Med 2008;358:111-124.

6. Hotchkiss RS, Monneret G, Payen D. Sepsis-induced immunosuppression: from cellular dysfunctions to immunotherapy. Nat Rev Immunol 2013;13:86274.

7. Bauer M, Giamarellos-Bourboulis EJ, Kortgen A, Möller E, Felsmann K, Cavaillon JM, Guntinas-Lichius O, Rutschmann O, Ruryk A, Kohl M, Wlotzka B, Rußwurm S, Marshall JC, Reinhart K. A Transcriptomic Biomarker to Quantify Systemic Inflammation in Sepsis - A Prospective Multicenter Phase II Diagnostic Study. EBioMedicine 2016;6:114-125.

8. Kaufmann SHE, Dorhoi A, Hotchkiss RS, Bartenschlager R. Host-directed therapies for bacterial and viral infections. Nat Rev Drug Discov 2017;17:3556.

9. Baschant U, Culemann S, Tuckermann J. Molecular determinants of glucocorticoid actions in inflammatory joint diseases. Mol Cell Endocrinol 2013;380:108-118.

10. Chinenov Y, Rogatsky I. Glucocorticoids and the innate immune system: crosstalk with the toll-like receptor signaling network. Mol Cell Endocrinol 2007;275:30-42.

11. Galon J, Franchimont D, Hiroi N, Frey G, Boettner A, Ehrhart-Bornstein M, O'Shea JJ, Chrousos GP, Bornstein SR. Gene profiling reveals unknown enhancing and suppressive actions of glucocorticoids on immune cells. FASEB J 2002;16:61-71.

12. Busillo JM, Azzam KM, Cidlowski JA. Glucocorticoids Sensitize the Innate Immune System through Regulation of the NLRP3 Inflammasome. J Biol Chem 2011;286:38703-38713.

13. Dhabhar FS. Stress-induced augmentation of immune function--the role of stress hormones, leukocyte trafficking, and cytokines. Brain Behav Immun 2002;16:785-798.

14. Cruz-Topete D, Cidlowski JA. One Hormone, Two Actions: Anti- and ProInflammatory Effects of Glucocorticoids. Neuroimmunomodulation 2015;22:2032.

15. Singer M, Deutschman CS, Seymour CW, Shankar-Hari M, Annane D, Bauer M, Bellomo R, Bernard GR, Chiche J-D, Coopersmith CM, Hotchkiss RS, Levy MM, Marshall JC, Martin GS, Opal SM, Rubenfeld GD, van der Poll T, Vincent $\mathrm{J}-\mathrm{L}$, Angus DC. The Third International Consensus Definitions for Sepsis and Septic Shock (Sepsis-3). JAMA 2016;315:801.

16. Giamarellos-Bourboulis EJ, Tsaganos T, Tsangaris I, Lada M, Routsi C, Sinapidis D, Koupetori M, Bristianou M, Adamis G, Mandragos K, Dalekos GN, Kritselis I, Giannikopoulos G, Koutelidakis I, Pavlaki M, Antoniadou E, Vlachogiannis G, Koulouras V, Prekates A, Dimopoulos G, Koutsoukou A, Pnevmatikos I, loakeimidou A, Kotanidou A, Orfanos SE, Armaganidis A, Gogos C, Hellenic Sepsis Study Group. Validation of the new Sepsis-3 definitions: proposal for improvement in early risk identification. Clin Microbiol 
Infect 2017;23:104-109.

17. Bloos F, Trips E, Nierhaus A, Briegel J, Heyland DK, Jaschinski U, Moerer O, Weyland A, Marx G, Gründling M, Kluge S, Kaufmann I, Ott K, Quintel M, Jelschen F, Meybohm P, Rademacher S, Meier-Hellmann A, Utzolino S, Kaisers UX, Putensen C, Elke G, Ragaller M, Gerlach H, Ludewig K, Kiehntopf $\mathrm{M}$, Bogatsch $\mathrm{H}$, Engel C, Brunkhorst FM, et al. Effect of Sodium Selenite Administration and Procalcitonin-Guided Therapy on Mortality in Patients With Severe Sepsis or Septic Shock. JAMA Intern Med 2016;176:1266.

18. Keh D, Boehnke T, Weber-Cartens S, Schulz C, Ahlers O, Bercker S, Volk HD, Doecke W-D, Falke KJ, Gerlach H. Immunologic and Hemodynamic Effects of "Low-Dose" Hydrocortisone in Septic Shock. Am J Respir Crit Care Med 2003;167:512-520.

19. Matera G, Puccio R, Giancotti A, Quirino A, Pulicari M, Zicca E, Caroleo S, Renzulli A, Liberto M, Focà A. Impact of interleukin-10, soluble CD25 and interferon- $y$ on the prognosis and early diagnosis of bacteremic systemic inflammatory response syndrome: a prospective observational study. Crit Care 2013;17:R64.

20. Medina TS, Costa SP, Oliveira MD, Ventura AM, Souza JM, Gomes TF, Vallinoto AC, Póvoa MM, Silva JS, Cunha MG. Increased interleukin-10 and interferon- $y$ levels in Plasmodium vivax malaria suggest a reciprocal regulation which is not altered by IL-10 gene promoter polymorphism. Malar $J$ 2011;10:264.

21. Djoba Siawaya JF, Beyers N, van Helden P, Walzl G. Differential cytokine secretion and early treatment response in patients with pulmonary tuberculosis. Clin Exp Immunol 2009;156:69-77.

22. Mcllleron H, Watkins ML, Folb PI, Ress SR, Wilkinson RJ. Rifampin levels, interferon-gamma release and outcome in complicated pulmonary tuberculosis. Tuberculosis 2007;87:557-564.

23. Chegou NN, Sutherland JS, Namuganga A-R, Corstjens PL, Geluk A, Gebremichael G, Mendy J, Malherbe S, Stanley K, van der Spuy GD, Kriel M, Loxton AG, Kriel B, Simukonda F, Bekele Y, Sheehama JA, Nelongo J, van der Vyver M, Gebrexabher A, Hailu H, Esterhuyse MM, Rosenkrands I, Aagard C, Kidd M, Kassa D, Mihret A, Howe R, Cliff JM, Crampin AC, et al. Africa-wide evaluation of host biomarkers in QuantiFERON supernatants for the diagnosis of pulmonary tuberculosis. Sci Rep 2018;8:2675.

24. Murray PJ, Wang L, Onufryk C, Tepper RI, Young RA. T cell-derived IL-10 antagonizes macrophage function in mycobacterial infection. $J$ Immunol 1997;158:315-321.

25. Jamil B, Shahid F, Hasan Z, Nasir N, Razzaki T, Dawood G, Hussain R. Interferon gamma/IL10 ratio defines the disease severity in pulmonary and extra pulmonary tuberculosis. Tuberculosis 2007;87:279-287.

26. Casserly B, Phillips GS, Schorr C, Dellinger RP, Townsend SR, Osborn TM, Reinhart K, Selvakumar N, Levy MM. Lactate Measurements in SepsisInduced Tissue Hypoperfusion. Crit Care Med 2015;43:567-573.

27. Schoenborn JR, Wilson CB. Regulation of Interferon- $y$ During Innate and Adaptive Immune Responses. Adv Immunol Academic Press, United States; 2007. p. 41-101.

28. Sabat R, Grütz G, Warszawska K, Kirsch S, Witte E, Wolk K, Geginat J. Biology of interleukin-10. Cytokine Growth Factor Rev 2010;21:331-344. 
29. Kühn R, Löhler J, Rennick D, Rajewsky K, Müller W. Interleukin-10-deficient mice develop chronic enterocolitis. Cell 1993;75:263-274.

30. Dellinger RP, Levy MM, Carlet JM, Bion J, Parker MM, Jaeschke R, Reinhart K, Angus DC, Brun-Buisson C, Beale R, Calandra T, Dhainaut J-F, Gerlach H, Harvey M, Marini JJ, Marshall J, Ranieri M, Ramsay G, Sevransky J, Thompson BT, Townsend S, Vender JS, Zimmerman JL, Vincent J-L, International Surviving Sepsis Campaign Guidelines Committee, American Association of Critical-Care Nurses, American College of Chest Physicians, American College of Emergency Physicians, Canadian Critical Care Society, et al. Surviving Sepsis Campaign: International guidelines for management of severe sepsis and septic shock: 2008. Crit Care Med 2008;36:296-327.

31. Dellinger RP, Levy MM, Rhodes A, Annane D, Gerlach H, Opal SM, Sevransky JE, Sprung CL, Douglas IS, Jaeschke R, Osborn TM, Nunnally ME, Townsend SR, Reinhart K, Kleinpell RM, Angus DC, Deutschman CS, Machado FR, Rubenfeld GD, Webb SA, Beale RJ, Vincent J-L, Moreno R, Surviving Sepsis Campaign Guidelines Committee including the Pediatric Subgroup. Surviving Sepsis Campaign. Crit Care Med 2013;41:580-637.

32. Bentzer P, Fjell C, Walley KR, Boyd J, Russell JA. Plasma cytokine levels predict response to corticosteroids in septic shock. Intensive Care Med 2016;42:1970-1979.

\section{Main Legends}

Table 1. Patient characteristics of the studied CORTICUS sub-cohort

Table 2. Survival rates according to high and low IFNY/LL10

Figure 1. Pie chart and Forest plot for outcome in CORTICUS and HSSG cohorts depending on hydrocortisone administration according to the theranostic marker (a) Survivors (red to light red) and non-survivors (black to grey) of all patients (left) and of patients being in compliance with the decision rule (right), patient numbers are given in the pies. Areas are proportional to number of patients. Treatment according to the decision rule was associated with an absolute risk reduction of 0.14 [95\% Cl: 0.03-0.26]. (b) Forest plot of the odds ratios for the sub-cohorts of CORTICUS (discovery set), HSSG (validation set) regarding IFNY/LL10 prediction rule, SISPCT, the Crossover study, the combination of all cohorts, as well as the ACTH test (for CORTICUS subcohort) are given (details are provided in Supplementary Text S10). For SISPCT a pseudo-count was added to each field preventing division by zero. 
Figure 2. Time courses of serum lactate and norepinephrine consumption depending on hydrocortisone administration and IFN/IL10 ratio.

(a) - (d) Normalized lactate levels, i.e. values for days 1-3 relative to day 0 for each patient are presented. Absolute serum lactate levels and p-values for the change over time in all available patients of the corresponding sub-group are reported in each panel. Patients in panels (a) and (d) reflect those in compliance with the treatment rule. For patients with high IFNY/IL10, serum lactate decreased significantly in the placebo arm $(P<0.01 n=11)(a)$, while its time course was rather heterogenous in the $H C$ arm $(n=13)(b)$. Among the low-ratio patients, there was a tendency of decrease in the placebo arm, however, not significant $(n=12)(c)$, in contrast to a significant decrease in the $H C$ arm $(P<0.01, n=5)(d)$.

(e) - (h) Norepinephrine consumption for days 1-3 is presented. Patients in panels (e) and (h) reflect those in compliance with the treatment rule. For patients with high IFNY/LL10, NE consumption decreased significantly in the placebo arm $(P<0.01 n=21)(e)$, while its time course was rather heterogenous in the $\mathrm{HC}$ arm $(n=29)(f)$. Among the low-ratio patients, there was a heterogeneous trend in the placebo arm $(n=20)(g)$, in contrast to a significant decrease in the $\mathrm{HC}$ arm $(\mathrm{P}<0.01, \mathrm{n}=21)(\mathrm{h})$. Putting the compliant arms together (e and $\mathrm{h})$, the rate of decrease over time was highly significant $(p<2 e-05)$, but not significant in the non-complaint arm (panel f) and g), $\mathrm{p}>0.5$ ).

Figure 3. IFNy/IL10 reflects the immunological load of immune cells ex vivo. Whole blood from five healthy donors was challenged with varying dilutions of $E$. coli fragments mimicking the bacterial burden on the immune system in sepsis. IFNY (a) and IL10 (b) were elevated with increasing bacterial load while their ratio (c) showed the opposite behaviour, i.e. a high load was associated with a lower ratio.

\section{List of supplementary material}

The supplementary material is compiled in one file denoted as Supplementary material. 
Table 1. Patient characteristics of the studied CORTICUS sub-cohort

\begin{tabular}{lll}
\hline & $\begin{array}{l}\mathrm{HC} \\
(\mathrm{n}=42)\end{array}$ & $\begin{array}{l}\text { Placebo } \\
(\mathrm{n}=41)\end{array}$ \\
\hline Sex (male/ female, $\mathrm{n})$ & $29 / 13$ & $30 / 11$ \\
Age [years] & $59.4(22-87)^{*}$ & $69.4(43-88)$ \\
Height [cm] & $171(150-188)$ & $172(150-195)$ \\
Weight [kg] & $79.5(50-130)$ & $77.1(53-127)$ \\
\hline Admission category (n) & & \\
- Medical & 1 & 0 \\
- Emergency surgery & 39 & 37 \\
- Elective surgery & 2 & 4 \\
\hline SOFA score at inclusion & $10.4(4-18)$ & $9.8(5-17)$ \\
SAPS II score & & \\
- First 24 hours at ICU & $47.9(22-80)$ & $48.0(16-88)$ \\
- Last 24 hours before inclusion & $43.7(13-77)$ & $47.2(16-88)$ \\
\hline Time: sepsis -> inclusion [hours] & $29(3-71)$ & $31(1-67)$ \\
\hline ACTH Responder/ Non-Responder (n) & $26 / 16$ & $29 / 12$ \\
\hline Survival (n) & & \\
- Day 28 (survivors/ non-survivors) & $31 / 11$ & $29 / 12$ \\
- ICU (survivors/ non-survivors) & $27 / 15$ & $28 / 13$ \\
- Hospital (survivors/ non-survivors) & $23 / 17$ & $26 / 15$ \\
\hline ICU- stay [days] all patients & $27.5(3-160)$ & $23.9(3-89)$ \\
ICU- stay [days] survivors & $23.3(5-73)$ & $26.3(3-89)$ \\
Hospital stay [days] all patients & $51.3(11-207)$ & $45.5(3-135)$ \\
Hospital stay [days] survivors & $50.5(15-128)$ & $53.9(17-135)$ \\
\hline
\end{tabular}

${ }^{*}: p<0.05 ;$ Range : minimum and maximum 
Table 2. Survival rates according to high and low IFNץ/IL10

a) CORTICUS patients treated with placebo

\begin{tabular}{lccc}
\hline & Non-survivors & Survivors & $\%$ Survivors $^{*}$ \\
\hline IFNy/IL10 high & 4 & 20 & $\mathbf{8 3 \%}{ }^{*}$ \\
IFNy/IL10 low & 8 & 9 & $\mathbf{5 3 \% 区}$ \\
\hline
\end{tabular}

b) CORTICUS patients treated with HC (hydrocortisone)

\begin{tabular}{lccc}
\hline & Non-survivors & Survivors & $\%$ Survivors \\
\hline IFNy/IL10 high & 9 & 20 & $\mathbf{6 9 \%}$ 区 \\
IFNy/IL10 low & 2 & 11 & $\mathbf{8 5 \%}$ \\
\hline
\end{tabular}

c) HSSG patients, not treated with HC

\begin{tabular}{lccc}
\hline & Non-survivors & Survivors & $\%$ Survivors \\
\hline IFNy/IL10 high & 27 & 27 & $\mathbf{5 0 \% \square}$ \\
IFNy/IL10 low & 32 & 13 & $\mathbf{2 9 \%} \square$ \\
\hline
\end{tabular}

d) HSSG patients, treated with HC

\begin{tabular}{lccc}
\hline & Non-survivors & Survivors & $\%$ Survivors \\
\hline IFNy/IL10 high & 21 & 8 & $\mathbf{2 8 \%}$ 凶 \\
IFNy/IL10 low & 22 & 12 & $\mathbf{3 5 \%} \square$ \\
\hline
\end{tabular}

e) SISPCT patients, not treated with HC

\begin{tabular}{lccc}
\hline & Non-survivors & Survivors & $\%$ Survivors \\
\hline IFNy/IL10 high & 0 & 8 & $\mathbf{1 0 0 \% \square}$ \\
IFNy/IL10 low & 0 & 6 & $\mathbf{1 0 0 \%} \square$ \\
\hline
\end{tabular}

f) SISPCT patients, treated with $\mathrm{HC}$

\begin{tabular}{lccc}
\hline & Non-survivors & Survivors & $\%$ Survivors \\
\hline IFNy/IL10 high & 3 & 4 & $\mathbf{5 7 \%}$ 凶 \\
IFNy/L10 low & 0 & 3 & $\mathbf{1 0 0 \%}$ \\
\hline
\end{tabular}

g) Patients from the early arm of the Crossover study (treated with $\mathrm{HC}$ similar to the CORTICUS HC arm)

\begin{tabular}{lccc}
\hline & Non-survivors & Survivors & $\%$ Survivors \\
\hline IFNy/IL10 high & 5 & 7 & $\mathbf{5 4 \%}$ 区 \\
\hline IFNy/IL10 low & 1 & 7 & $\mathbf{8 8 \%}$ \\
\hline
\end{tabular}

h) Confusion matrices and survival rates when combining the results from the three studies 


\begin{tabular}{cccc}
\hline All studies & $\begin{array}{c}\text { Non- } \\
\text { survivors }\end{array}$ & Survivors & $\%$ Survivors \\
\hline $\begin{array}{c}\text { Patients in compliance } \\
\text { with our rule } \\
\begin{array}{c}\text { Patients not in } \\
\text { compliance with our }\end{array}\end{array}$ & 56 & 88 & $61 \%$ \\
$\begin{array}{c}\text { rule } \\
\text { All patients }\end{array}$ & 134 & 155 & $56 \%$ \\
\hline
\end{tabular}

${ }^{\star} \nabla$ and $\otimes$ indicate $\mathrm{HC}$ treatment in compliance and not in compliance with our decision rule, respectively 


\section{Figure 1}

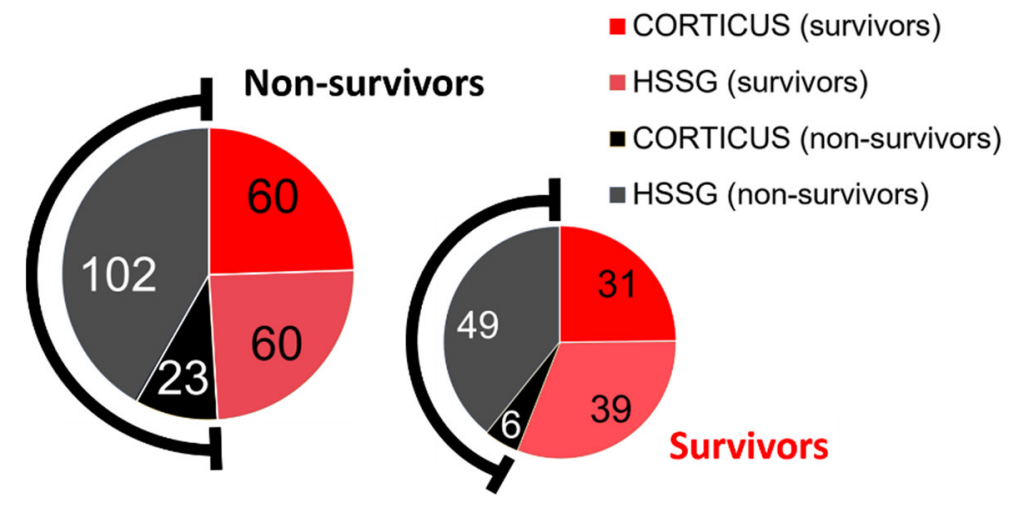

a)

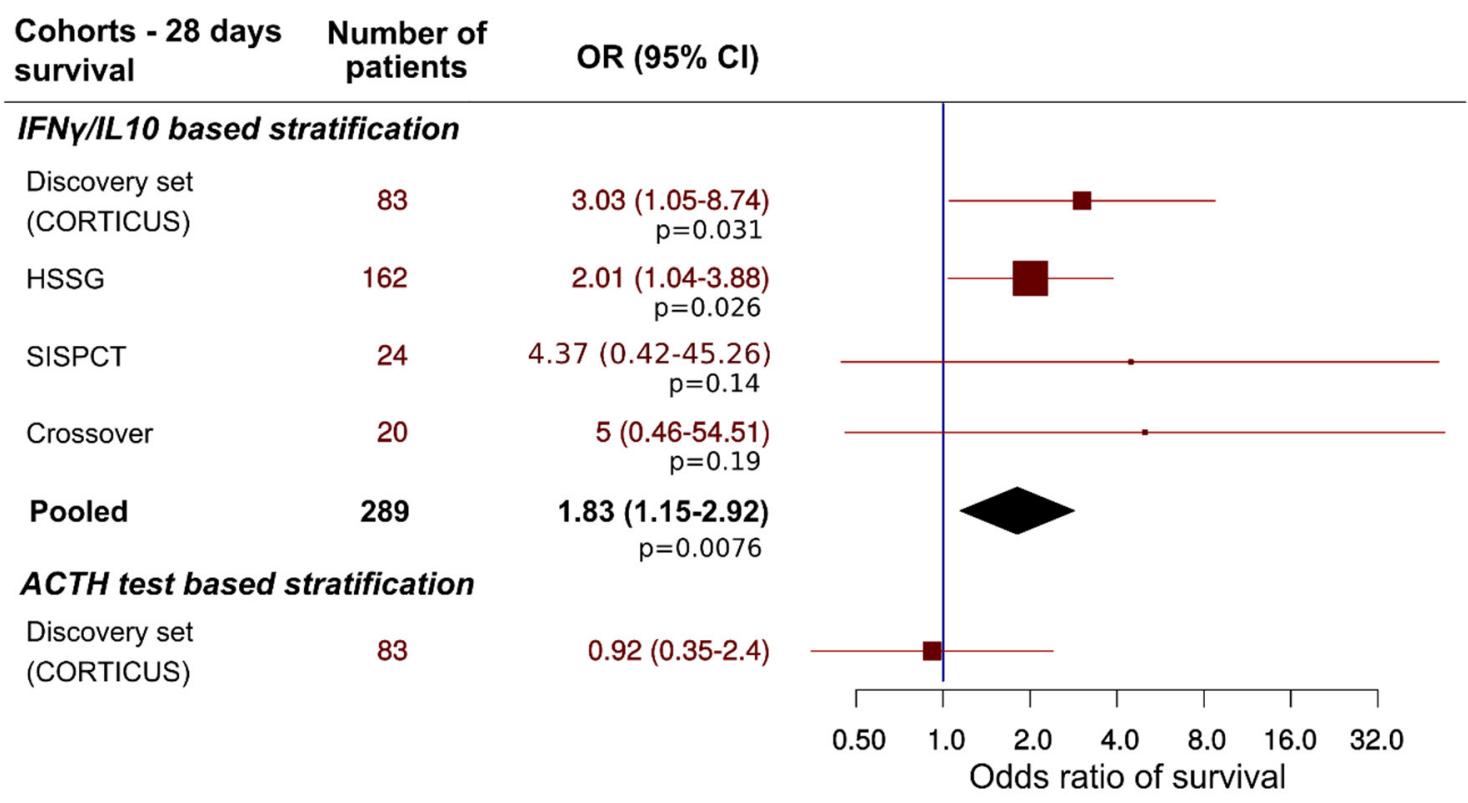

b) 


\section{Figure 2}
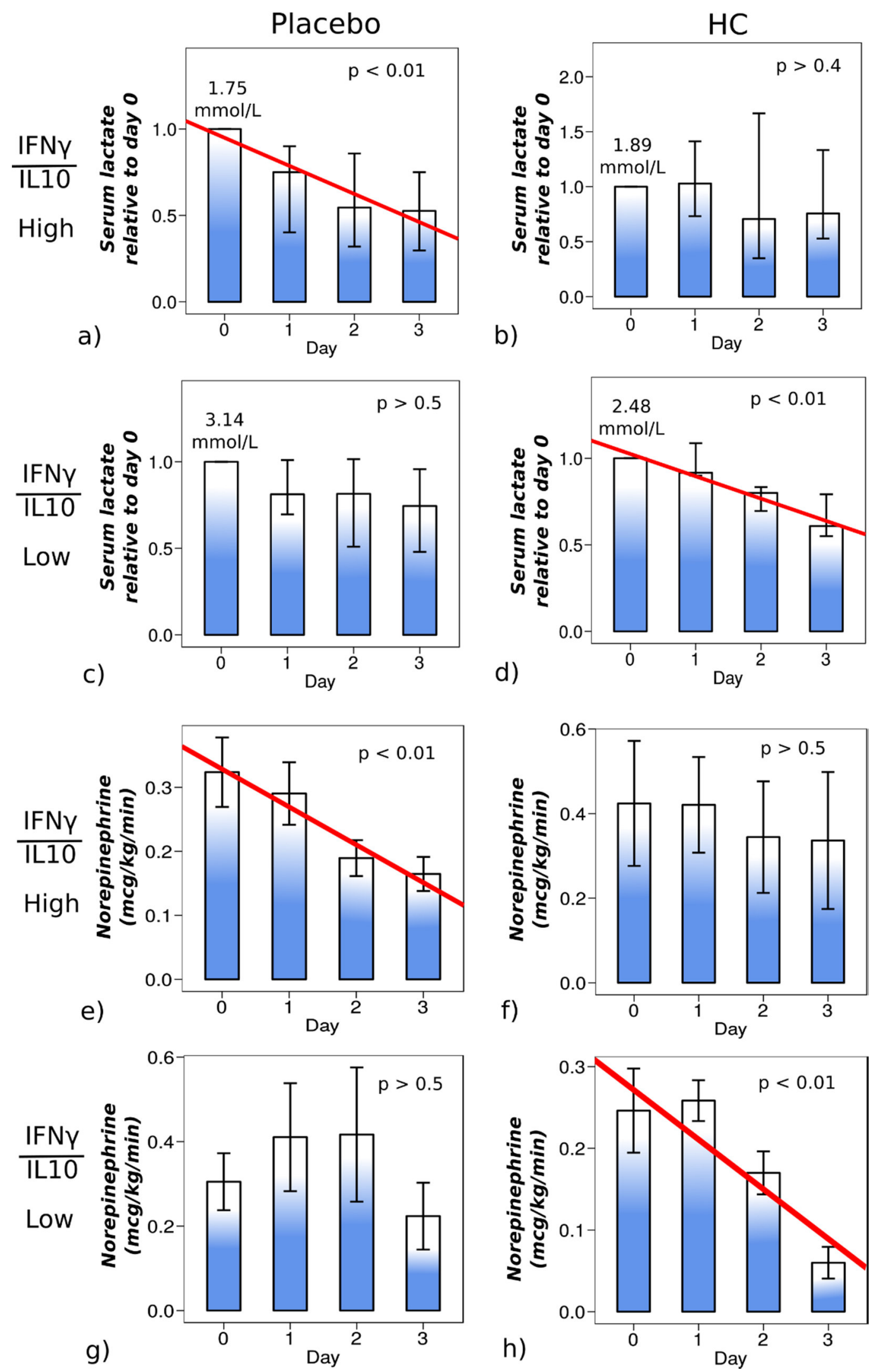


\section{Figure 3}

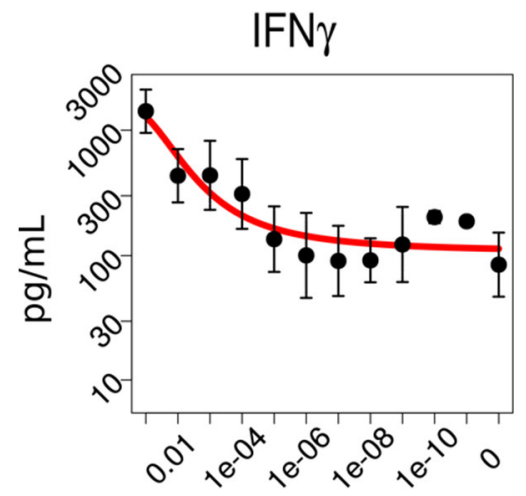

a) E. coli fragments

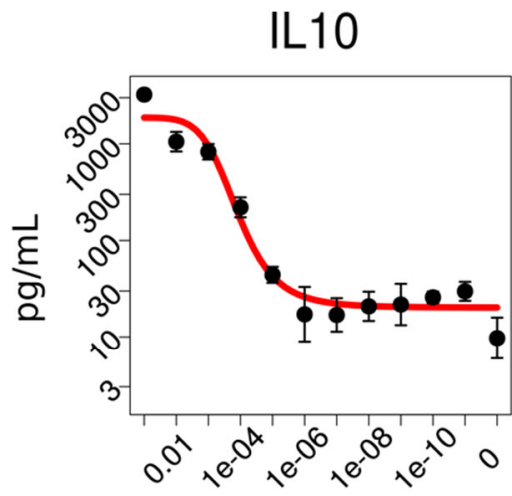

b) (serial dilutions)
IFN $\gamma /$ IL 10

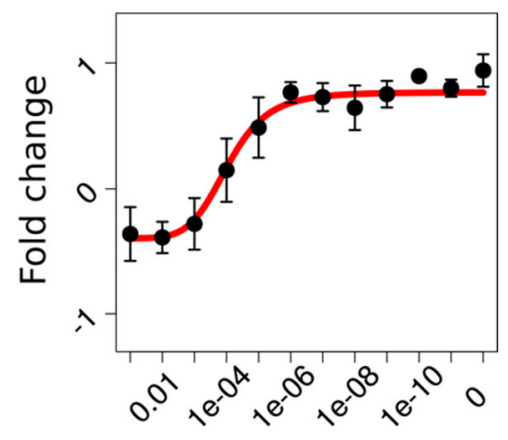

c)
E. coli fragments (serial dilutions)

_ Fit using the mean 